Proyecciones Journal of Mathematics

Vol. 35, No 2, pp. 213-223, June 2016.

Universidad Católica del Norte

Antofagasta - Chile

\title{
On Jensen's and the quadratic functional equations with involutions
}

\author{
B. Fadli \\ A. Chahbi \\ Iz. El-Fassi \\ and \\ S. Kabbaj \\ IBN Tofail University, Morocco \\ Received : March 2016. Accepted : May 2016
}

\begin{abstract}
We determine the solutions $f: S \rightarrow H$ of the generalized Jensen's functional equation

$$
f(x+\sigma(y))+f(x+\tau(y))=2 f(x), \quad x, y \in S,
$$

and the solutions $f: S \rightarrow H$ of the generalized quadratic functional equation

$$
f(x+\sigma(y))+f(x+\tau(y))=2 f(x)+2 f(y), \quad x, y \in S,
$$

where $S$ is a commutative semigroup, $H$ is an abelian group (2-torsion free in the first equation and uniquely 2-divisible in the second) and $\sigma, \tau$ are two involutions of $S$.
\end{abstract}

Subjclass [2010] : Primary 39B52.

Keywords : Functional equation, Jensen, quadratic, additive function, semigroup. 


\section{Set up, notation and terminology}

Throughout the paper we work in the following framework and with the following notation and terminology. We use it without explicit mentioning. $S$ is a commutative semigroup [a set equipped with an associative composition rule $(x, y) \mapsto x+y], \sigma, \tau: S \rightarrow S$ are two homomorphisms satisfying $\sigma \circ \sigma=\tau \circ \tau=i d$, and $(H,+)$ denotes an abelian group with neutral element 0 . We say that $H$ is 2 -torsion free if $[h \in H$ and $2 h=0] \Rightarrow h=0$.

$H$ is said to be uniquely 2-divisible if for any $h \in H$ the equation $2 x=h$ has exactly one solution $x \in H$.

A function $A: S \rightarrow H$ is said to be additive if $A(x+y)=A(x)+A(y)$ for all $x, y \in S$.

We recall that the Cauchy difference $C f$ of a function $f: S \rightarrow H$ is defined by

$$
C f(x, y):=f(x+y)-f(x)-f(y), \quad x, y \in S .
$$

\section{Introduction}

In [16], Sinopoulos determined the general solution $f: S \rightarrow H$, where $H$ is 2-torsion free, of Jensen's functional equation

$$
f(x+y)+f(x+\tau(y))=2 f(x), \quad x, y \in S,
$$

and the general solution $f: S \rightarrow H$, where $H$ is uniquely 2-divisible, of the quadratic functional equation

$$
f(x+y)+f(x+\tau(y))=2 f(x)+2 f(y), \quad x, y \in S .
$$

Some information, applications and numerous references concerning (2.1) and (2.2) and their further generalizations can be found, e.g., in [3-8, 10-14, $17,18]$. For more details, we refer to the monographs $[9,15,19]$.

The purpose of the present paper is to solve the following functional equations

$$
\begin{gathered}
f(x+\sigma(y))+f(x+\tau(y))=2 f(x), \quad x, y \in S, \\
f(x+\sigma(y))+f(x+\tau(y))=2 f(x)+2 f(y), \quad x, y \in S .
\end{gathered}
$$

Thus the contribution by our paper of new knowledge consists in introducing an involution $\sigma$ and in solving the corresponding extensions (2.3) and 
(2.4) of the functional equations (2.1) and (2.2). Our solution formulas contain the previous ones as special cases.

A similar functional equation that has been studied is

$$
f(x+\sigma(y))+f(x+\tau(y))=2 f(x) f(y), \quad x, y \in S,
$$

where $f: S \rightarrow \mathbf{C}$ is the function to determine. Eq. (2.5) was solved in a more general framework (see [2]).

\section{On Jensen's functional equation}

In this section, we solve the functional equation (2.3) by expressing its solutions in terms of additive functions.

Lemma 3.1. Let $f: S \rightarrow H$ be a solution of the functional equation (2.3). Then

$$
f\left(x+(\tau \circ \sigma)^{2}(y)\right)=f(x+y) \quad \text { for all } x, y \in S .
$$

Proof. Making the substitutions $(x, \sigma(y))$ and $(x, \tau(y))$ in $(2.3)$, we get respectively

$$
\begin{aligned}
& f(x+y)+f(x+\tau(\sigma(y)))=2 f(x) \\
& f(x+\sigma(\tau(y)))+f(x+y)=2 f(x) .
\end{aligned}
$$

So

$$
f(x+\tau(\sigma(y)))=f(x+\sigma(\tau(y))) \quad \text { for all } x, y \in S .
$$

Replacing here $y$ by $\tau(\sigma(y))$, we obtain (3.1).

Theorem 3.2. Suppose that $H$ is 2-torsion free. The general solution $f: S \rightarrow H$ of the functional equation (2.3) is $f=A+c$, where $A: S \rightarrow H$ is an additive map such that $A \circ \tau=-A \circ \sigma$, and where $c \in H$ is a constant.

Proof. The method used here is closely related to and inspired by the one in [16, Proof of Theorem 2]. Assume that $f: S \rightarrow H$ is a solution of (2.3). Then

$$
f(x+y)+f(x+\tau(\sigma(y)))=2 f(x), \quad x, y \in S .
$$


Making the substitutions $(x, y+\tau(\sigma(y)))$ and $(x+z, y)$ in $(3.2)$ and using Lemma 3.1, we get respectively

$$
\begin{gathered}
f(x+y+\tau(\sigma(y)))=f(x), \\
f(x+z+y)+f(x+z+\tau(\sigma(y)))=2 f(x+z) .
\end{gathered}
$$

Interchanging $y$ and $z$ in the last equation we have

$$
f(x+y+z)+f(x+y+\tau(\sigma(z)))=2 f(x+y) .
$$

Adding the last two equations we obtain

$$
\begin{gathered}
2 f(x+y+z)+f(x+y+\tau(\sigma(z)))+f(x+z+\tau(\sigma(y))) \\
=2 f(x+z)+2 f(x+y) .
\end{gathered}
$$

Using Lemma 3.1, we get that

$$
f(x+z+\tau(\sigma(y)))=f(x+(\tau \circ \sigma)[y+(\tau \circ \sigma)(z)]) .
$$

So, using (3.2), we can reformulate (3.4) to

$$
2 f(x+y+z)+2 f(x)=2 f(x+z)+2 f(x+y) .
$$

Setting here $z=\tau(\sigma(x))$ and using (3.3) and the fact that $H$ is 2-torsion free, we get

$$
f(y)+f(x)=f(x+\tau(\sigma(x)))+f(x+y) .
$$

Interchanging $x$ and $y$ in (3.5), we get that

$$
f(x+\tau(\sigma(x)))=f(y+\tau(\sigma(y)))
$$

for all $x, y \in S$. So $f(x+\tau(\sigma(x)))$ is a constant, say $c$. By using (3.5), we infer that the function $A(x):=f(x)-c$ is additive. Substituting $f$ into (2.3) we see that $A \circ \tau=-A \circ \sigma$.

The other direction of the proof is trivial to verify.

As a immediate consequence of Theorem 3.2, we have the following result.

Corollary 3.3. [16, Theorem 2] Suppose that $H$ is 2-torsion free. The general solution $f: S \rightarrow H$ of the functional equation (2.1) is $f=A+c$, where $A: S \rightarrow H$ is an additive map such that $A \circ \tau=-A$, and where $c \in H$ is a constant. 


\section{On the quadratic functional equation}

In this section, we generalize Sinopoulos's result [16, Theorem 3] on semigroups by solving the functional equation (2.4). The following lemma lists pertinent basic properties of any solution $f: S \rightarrow H$ of (2.4).

Lemma 4.1. Suppose that $H$ is 2-torsion free and let $f: S \rightarrow H$ be a solution of the functional equation (2.4).

a) $f \circ \sigma+f \circ \tau=2 f$.

b) Let $A: S \rightarrow H$ be $A:=f \circ \sigma-f \circ \tau$. Then $A$ is additive and $A \circ \sigma=A \circ \tau=-A$.

c) For all $x, y, z \in S$, we have

$$
f(x+y+z)=f(x+y)+f(x+z)+f(y+z)-f(x)-f(y)-f(z) .
$$

d) $C f: S \times S \rightarrow H$ is a symmetric, bi-additive map satisfying

$C f(x, \tau(y))=-C f(x, \sigma(y))$ for all $x, y \in S$.

e) Let $\varphi: S \rightarrow H$ be $\varphi(x):=A(x)+2 f(x+\tau(x)), x \in S$. Then $\varphi \circ \sigma+\varphi \circ \tau=2 \varphi$ and $\varphi$ satisfies that

$$
\varphi(x+y)=\varphi(x)+\varphi(y)+4\{C f(x, y)-C f(x, \sigma(y))\}, \quad x, y \in S .
$$

f) $\varphi$ is a solution of (2.4).

g) $4 f(x)=2 C f(x, \sigma(x))+\varphi(x)$ for all $x \in S$.

Proof. (a) Let us first observe that $f \circ \sigma+f \circ \tau$ is a solution of (2.4). We next replace $x$, first by $\sigma(x)$ and then by $\tau(x)$, in (2.4) we find that

$$
\begin{aligned}
& f(\sigma(x)+\sigma(y))+f(\sigma(x)+\tau(y))=2 f(\sigma(x))+2 f(y), \\
& f(\tau(x)+\sigma(y))+f(\tau(x)+\tau(y))=2 f(\tau(x))+2 f(y) .
\end{aligned}
$$

Summing these two equations and using (2.4) and the fact that $H$ is 2-torsion free, we obtain

$$
[f(x)+f(\sigma(y))]+[f(x)+f(\tau(y))]=f(\sigma(x))+f(\tau(x))+2 f(y),
$$

i.e.

$$
2 f(x)-f(\sigma(x))-f(\tau(x))=2 f(y)-f(\sigma(y)-f(\tau(y)),
$$


for all $x, y \in S$. From this last equation we infer that $2 f-f \circ \sigma-f \circ \tau$ is a constant in $H$, say $c$. Using the fact that $2 f-(f \circ \sigma+f \circ \tau)$ is a solution of (2.4) and that $H$ is 2-torsion free, we see that $c=0$.

(b) We subtract (4.3) from (4.2) and get that

$$
(f \circ \sigma-f \circ \tau)(x+y)+[f(\sigma(x)+\tau(y))-f(\tau(x)+\sigma(y))]=2(f \circ \sigma-f \circ \tau)(x),
$$

for all $x, y \in S$. By using (2.4) and (a), we have

$$
\begin{aligned}
f(\sigma(x)+\tau(y))-f(\tau(x)+\sigma(y)) \\
\quad=[f(\sigma(x)+\tau(y))+f(\tau(x)+\tau(y))]-2 f \circ \tau(x)-2 f(y) \\
\quad=2 f(x)+2 f \circ \tau(y)-2 f \circ \tau(x)-2 f(y) \\
\quad=(f \circ \sigma-f \circ \tau)(x)-(f \circ \sigma-f \circ \tau)(y),
\end{aligned}
$$

which turns the identity (4.4) into

$$
(f \circ \sigma-f \circ \tau)(x+y)=(f \circ \sigma-f \circ \tau)(x)+(f \circ \sigma-f \circ \tau)(y),
$$

for all $x, y \in S$. This show that the function $A=f \circ \sigma-f \circ \tau$ is additive. Using $(a)$, we see that

$$
A=2 f-2 f \circ \tau=2 f \circ \sigma-2 f .
$$

So, $A \circ \sigma=A \circ \tau=-A$.

(c) Making the substitutions $(x+y, \sigma(z)),(x+\tau(\sigma(z)), \sigma(y))$, and $(x, \sigma(y+$ $z)$ ) in (2.4), we get respectively

$$
\begin{aligned}
& f(x+y+z)+f(x+y+\tau(\sigma(z)))=2 f(x+y)+2 f(\sigma(z)), \\
& f(x+\tau(\sigma(z))+y)+f(x+\tau(\sigma(y+z)))=2 f(x+\tau(\sigma(z))+2 f(\sigma(y)) \\
& =2[2 f(x)+2 f(\sigma(z))-f(x+z)]+2 f(\sigma(y)), \\
& f(x+y+z)+f(x+\tau(\sigma(y+z)))=2 f(x)+2 f(\sigma(y+z)) .
\end{aligned}
$$

Subtracting the middle identity from the sum of the other two we get that

$$
\begin{aligned}
2 f(x+y+z)= & 2 f(x+y)+2 f(x+z)+2 f(\sigma(y+z)) \\
& -2 f(x)-2 f(\sigma(y))-2 f(\sigma(z)) .
\end{aligned}
$$

Replacing here $2 f \circ \sigma$ by $2 f+A$ and using the fact that $H$ is 2 -torsion free, we get (4.1). 
(d) That $C f$ is symmetric and bi-additive follows immediately from the very definition of $C f$ and (4.1). Let $x, y \in S$ be arbitrary. By help of (4.1) and $(a)$, we get that

$$
\begin{aligned}
C f(x, \tau(y)) & =f(x+\tau(y))-f(x)-f(\tau(y)) \\
& =2 f(x)+2 f(y)-f(x+\sigma(y))-f(x)-f(\tau(y)) \\
& =f(x)+f(\sigma(y))-f(x+\sigma(y)) \\
& =-C f(x, \sigma(y)) .
\end{aligned}
$$

(e) For all $x \in S$, we have

$$
\begin{aligned}
(\varphi \circ & \sigma+\varphi \circ \tau)(x) \\
& =A(\sigma(x))+2 f(\sigma(x)+\tau(\sigma(x)))+A(\tau(x))+2 f(\tau(x)+x) \\
& =-2 A(x)+2 f(x+\tau(x))+8 f(\sigma(x))-2 f(x+\sigma(x)) \\
& =-2 A(x)+2 f(x+\tau(x))+8 f(x)+4 A(x)-2 f(x+\sigma(x)) \\
& =2 A(x)+4 f(x+\tau(x)) \\
& =2 \varphi(x) .
\end{aligned}
$$

So, $\varphi \circ \sigma+\varphi \circ \tau=2 \varphi$.

Next, let $x, y \in S$ be arbitrary. Using (4.1) repeatedly and the fact that $2 f \circ \tau=2 f-A$ and that $A \circ \tau=-A$ we find

$$
\begin{aligned}
& \varphi(x+y)=A(x+y)+2 f((x+\tau(x))+y+\tau(y)) \\
&= A(x)+A(y)+2 f(x+\tau(x)+y)+2 f(x+\tau(x)+\tau(y)) \\
&+2 f(y+\tau(y))-2 f(x+\tau(x))-2 f(y)-2 f(\tau(y)) \\
&= A(x)+A(y)+[2 f(x+\tau(x))+2 f(x+y)+2 f(\tau(x)+y) \\
&-2 f(x)-2 f(\tau(x))-2 f(y)]+[2 f(x+\tau(x))+2 f(x+\tau(y)) \\
&+2 f \circ \tau(x+y)-2 f(x)-2 f(\tau(x))-2 f(\tau(y))] \\
&+2 f(y+\tau(y))-2 f(x+\tau(x))-2 f(y)-2 f(\tau(y)) \\
&= \varphi(x)+\varphi(y)+[2 f(x+y)+2 f \circ \tau(x+y)]+[2 f(x+\tau(y)) \\
&+2 f \circ \tau(x+\tau(y))]-4 f(x)-4 f \circ \tau(x)-4 f(y)-4 f \circ \tau(y) \\
&= \varphi(x)+\varphi(y)+4 f(x+y)+4 f(x+\tau(y))-A(x+y) \\
&-A(x+\tau(y))-8 f(x)+2 A(x)-4 f(y)-4 f \circ \tau(y) \\
&= \varphi(x)+\varphi(y)+4 C f(x, y)+4 C f(x, \tau(y)) \\
&= \varphi(x)+\varphi(y)+4\{C f(x, y)-C f(x, \sigma(y))\} .
\end{aligned}
$$


(f) Using $(e)$ and $(d)$, we get

$$
\begin{aligned}
\varphi(x+ & \sigma(y))+\varphi(x+\tau(y)) \\
= & 2 \varphi(x)+\varphi(\sigma(y))+\varphi(\tau(y))+4\{C f(x, \sigma(y))-C f(x, y)\} \\
& +4\{C f(x, \tau(y))-C f(x, \sigma(\tau(y)))\} \\
= & 2 \varphi(x)+2 \varphi(y)+4\{C f(x, \sigma(y))-C f(x, y)\} \\
& +4\{-C f(x, \sigma(y))+C f(x, y)\} \\
= & 2 \varphi(x)+2 \varphi(y) .
\end{aligned}
$$

So, $\varphi$ is a solution of $(2.4)$.

$(g)$ Using the equality $A=2 f \circ \sigma-2 f$ and (2.4), we obtain

$$
\begin{aligned}
2 C f & (x, \sigma(x))+\varphi(x) \\
= & 2 f(x+\sigma(x))-2 f(x)-2 f(\sigma(x))+A(x)+2 f(x+\tau(x)) \\
= & {[2 f(x+\sigma(x))+2 f(x+\tau(x))]-2 f(x)-2 f(\sigma(x))+2 f(\sigma(x)) } \\
& -2 f(x) \\
= & 8 f(x)-4 f(x) \\
= & 4 f(x) \text { for all } x \in S .
\end{aligned}
$$

The second main theorem of the present paper reads as follows.

Theorem 4.2. Suppose that $H$ is uniquely 2-divisible. The general solution $f: S \rightarrow H$ of the functional equation (2.4) is

$$
f(x)=Q(x, \sigma(x))+\psi(x), \quad x \in S,
$$

where $Q: S \times S \rightarrow H$ is an arbitrary symmetric, bi-additive map such that $Q(x, \tau(y))=-Q(x, \sigma(y))$ for all $x, y \in S$, and where $\psi: S \rightarrow H$ is an arbitrary solution of

$$
\psi(x+y)=\psi(x)+\psi(y)+2\{Q(x, y)-Q(x, \sigma(y))\}, \quad x, y \in S,
$$

such that $\psi \circ \sigma+\psi \circ \tau=2 \psi$.

Proof. That all solutions of (2.4) have this form is a consequence of Lemma 4.1 and the fact that $H$ is uniquely 2-divisible. Conversely, simple computations based on the properties of $Q$ and $\psi$, show that the indicated functions are solutions.

As a immediate consequence of Theorem 4.2, we have the following result. 
Corollary 4.2. [16, Theorem 3] Suppose that $H$ is uniquely 2-divisible. The general solution $f: S \rightarrow H$ of the functional equation (2.2) is

$$
f(x)=Q(x, x)+\psi(x),
$$

where $Q: S \times S \rightarrow H$ is an arbitrary symmetric, bi-additive map such that

$Q(x, \tau(y))=-Q(x, y)$ for all $x, y \in S$, and where $\psi: S \rightarrow H$ is an arbitrary additive map such that $\psi \circ \tau=\psi$.

\section{References}

[1] J. Aczél and J. Dhombres, Functional equations in several variables, Cambridge University Press, New York (1989).

[2] A. Chahbi, B. Fadli, S. Kabbaj, A generalization of the symmetrized multiplicative Cauchy equation, Acta Math. Hungar., pp. 1-7, (2016).

[3] J. K. Chung, B. R. Ebanks, C. T. Ng and P. K. Sahoo, On a quadratic trigonometric functional equation and some applications, Trans. Amer. Math. Soc., 347, pp. 1131-1161, (1995).

[4] B. Fadli, D. Zeglami and S. Kabbaj, On a Gajda's type quadratic equation on a locally compact abelian group, Indagationes Math., 26, pp. 660-668, (2015).

[5] B. Fadli, D. Zeglami and S. Kabbaj, A variant of Jensen's functional equation on semigroups, Demonstratio Math., to appear.

[6] P. de Place Friis and H. Stetkær, On the quadratic functional equation on groups, Publ. Math. Debrecen 69, pp. 65-93, (2006).

[7] S-M. Jung, Quadratic functional equations of Pexider type, J. Math. \& Math. Sci., 24, pp. 351-359, (2000).

[8] P. Kannappan, Quadratic functional equation and inner product spaces, Results Math., 27, pp. 368-372, (1995).

[9] P. Kannappan, Functional equations and inequalities with applications, Springer, New York, (2009). 
[10] C. T. Ng, Jensen's functional equation on groups, Aequationes Math. 39, pp. 85-99, (1990).

[11] C. T. Ng, Jensen's functional equation on groups, II, Aequationes Math. 58, pp. 311-320, (1999).

[12] C. T. Ng, Jensen's functional equation on groups, III, Aequationes Math. 62, pp. 143-159, (2001).

[13] C. T. Ng, A Pexider-Jensen functional equation on groups, Aequationes Math. 70, pp. 131-153, (2005).

[14] J. C. Parnami and H.L. Vasudeva, On Jensen's functional equation, Aequationes Math. 43, pp. 211-218, (1992).

[15] Th. M. Rassias, Inner Product Spaces and Applications, Pitman Research Notes in Mathematics Series, Addison Wesley Longman Ltd, 376, (1997).

[16] P. Sinopoulos, Functional equations on semigroups, Aequationes Math., 59, pp. 255-261, (2000).

[17] H. Stetkær, Functional equations on abelian groups with involution, Aequationes Math. 54, pp. 144-172, (1997).

[18] H. Stetkær, On Jensen's functional equation on groups, Aequationes Math. 66, pp. 100-118, (2003).

[19] H. Stetkær, Functional Equations on Groups, World Scientific Publishing Co, Singapore, (2013).

\section{B. Fadli}

Department of Mathematics, Faculty of Sciences, Ibn Tofail University, B. P. 14000. Kenitra, Morocco

e-mail : himfadli@gmail.com 


\section{A. Chahbi}

Department of Mathematics,

Faculty of Sciences,

Ibn Tofail University,

B. P. 14000. Kenitra,

Morocco

e-mail : abdellatifchahbi@gmail.com

Iz. EL-Fassi

Department of Mathematics,

Faculty of Sciences,

Ibn Tofail University,

B. P. 14000. Kenitra,

Morocco

e-mail : izidd-math@hotmail.fr

and

S. Kabbaj

Department of Mathematics,

Faculty of Sciences,

Ibn Tofail University,

B. P. 14000. Kenitra,

Morocco

e-mail : samkabbaj@yahoo.fr 